\title{
Mesures et modèles conductifs
}

\section{Conductive measurements and models}

\author{
R. Gable, P. Watremez, B. Coleno
}

\author{
Laboratoire Potentiel géothermique national \\ Institut mixte de recherches géothermiques \\ (BRGM-AFME) - Orléans
}

Mesures et modèles thermiques sont combinés pour tendre vers une ébauche réaliste de la distribution du champ de température en bassins sédimentaires. Les résultats des travaux en cours, sur le Bassin parisien, sont présentés: - acquisition des données dans les forages géothermiques, - corrélation entre puits et synthèse des résultats.

- modèles de comportement thermique du sous-sol et interprétation des anomalies observées.
Thermal measurements and models are combined to attempt to achieve a realistic approach to temperature field distribution in sedimentation basins. The results of the works in progress in the Paris Basin are described

- date acquisition in geothermal drillings

- correlation between wells and synthesis of the results

- thermal behavior models of the subsoil and interpretation of the anomalies observed.

\section{Introduction}

Parmi les différents paramètres caractérisant les réservoirs géothermiques, les paramètres thermiques sont essentiels : la géothermie, méthode géophysique, est l'étude des lois qui régissent la distribution des températures en profondeur et des causes qui perturbent cette distribution. L'accroissement de la température avec la profondeur témoigne de l'existence d'un flux de chaleur se dissipant à la surface de la Terre. Des hypothèses variées sont formulées quant à son origine. On distingue :

- un flux d'origine profonde appelé classiquement flux réduit;

- un flux crustal résultant de la désintégration radioactive essentiellement des isotopes ${ }^{235} \mathrm{U},{ }^{238} \mathrm{U},{ }^{40} \mathrm{~K},{ }^{232} \mathrm{Th}$ des roches.
Il est difficile d'évaluer le pourcentage de chaleur radioactive entrant dans le flux total (mesuré en "surface "). Des estimations sont faites autour de 1/3,1/2. Des ordres de grandeur de cette source de chaleur peuvent être avancés compte tenu de l'énergie dégagée par chaque désintégration (Birch, 1968). L'incertitude demeure quant à la répartition de ces éléments radioactifs avec la profondeur. Différents modèles sont proposés (Lachenbruch, 1970; Allis, 1979; Smithson et Decker, 1979...).

Le flux de chaleur en surface, est $\Phi_{z}=-\mathrm{K}$ grad $\theta$, en supposant un transfert de chaleur vertical, dans un milieu homogène et isotrope. Sa détermination nécessite la connaissance du gradient géothermique et de la conduc- 
tivité thermique du milieu dans lequel se propage la chaleur. Ce dernier paramètre est surtout mesuré en laboratoire. Les recherches actuelles tendent vers une acquisition in situ, directe ou indirecte par méthode corrélative. La mesure de la température en forage ne pose techniquement aucun problème sérieux, mais elle nécessite une méthodologie spécifique. L'exploration géothermique a apporté les sites privilégiés qui manquaient jusqu'alors en permettant d'intervenir dans des puits profonds (plus de $1000 \mathrm{~m}$ ) après dissipation de la perturbation thermique résultant des conditions de forage. On devait auparavant se contenter des forages peu profonds.

\section{Les mesures thermiques en forage}

Elles constituent l'information de base essentielle dans la recherche sur l'état thermique actuel des bassins sédimentaires. Elles permettent l'interpolation entre puits et le choix de modèles réalistes de comportement thermique du sous-sol. Un effort tout particulier est donc consacré au développement des moyens d'investigation en forage : améliorer la qualité des mesures et diversifier l'acquisition. Des résultats sont obtenus sur ces deux points. Cette évolution peut être décrite de la manière suivante :

L'enregistrement de la température s'effectue de façon continue à la descente avec une vitesse lente (quelques mètres à la minute). De la mesure en paliers on a évolué vers la mesure en continu. Les variations du gradient géothermique peuvent être déduites de l'examen des changements de pente observés sur l'enregistrement (Figure 1). Cet exemple montre les variations rapides du gradient liées aux changements lithologiques. Il est faible (inférieur à $2^{\circ} \mathrm{C} / 100 \mathrm{~m}$ ) dans les formations salifères conductrices de la chaleur (Keuper). Le gradient géothermique moyen est de $3^{\circ} \mathrm{C} / 100 \mathrm{~m}$. Il est élevé dans les formations argileuses $\left(4^{\circ} \mathrm{C} / 100 \mathrm{~m}\right)$.

Une meilleure définition du gradient géothermique a été obtenue en digitalisant la courbe d'enregistrement en continu de la température (Figure 2). Cette configuration permet une meilleure analyse des variations de la température avec la profondeur ainsi que des corrélations plus fines entre diagraphies thermiques. Le pas de digitalisation de la diagraphie présentée est de 10 mètres. Ce profil du gradient géothermique obtenu souligne mieux les variations rapides de la pente du profil de température en fonction de la profondeur.

Maintenant grâce au développement des moyens techniques performants (utilisation d'un micro-ordinateur et élaboration de logiciels spécifiques de traitement), on est en mesure d'obtenir simultanément des enregistrements des profils de température et du gradient géothermique avec la profondeur.
Rappelons brièvement le principe de fonctionnement de l'unité d'acquisition de la température en forage que nous avons mis au point (Figure 3 ).

La température est enregistrée en continu, jusqu'à 3500 mètres de profondeur, avec une sensibilité de l'ordre du $1 / 100^{\circ}$ de degré et une température maximale de $200^{\circ} \mathrm{C}$. L'élément sensible utilisé est une thermistance. Le principe repose sur la mesure de la résistance (fonction de la température) d'une thermistance. On a un montage quatre fils utilisant :

- une source de courant étalon dont l'intensité de sortie est réglée de façon à ne pas échauffer la thermistance (dans notre cas : $100 \mu \mathrm{A}$ );

- un voltmètre numérique pour la mesure de la chute de tension aux bornes de la thermistance.

D'autres appareils annexes (contrôle, enregistrement,...) complètent ce dispositif de mesure.

En 1983, cette unité a été complétée par un microordinateur HP 85 permettant l'enregistrement des mesures sur cassettes magnétiques. La digitalisation a nécessité alors la conception de différents logiciels d'acquisition et de traitement de l'information.

Deux exemples de diagraphies thermiques prises dans des puits distants d'environ 50 kilomètres (Figure 4) soulignent :

- des corrélations significatives d'une diagraphie thermique à l'autre;

- des configurations thermiques spécifiques à chaque formation géologique (Crétacé par exemple).

De même les corrélations avec des diagraphies classiques (sonique, électrique) s'effectuent aisément (puits de Fontainebleau).

Les efforts tendent actuellement vers une évaluation en continu de la conductivité thermique in situ. Mais celà nécessite préliminairement la définition d'un certain nombre de paramètres correctifs. 


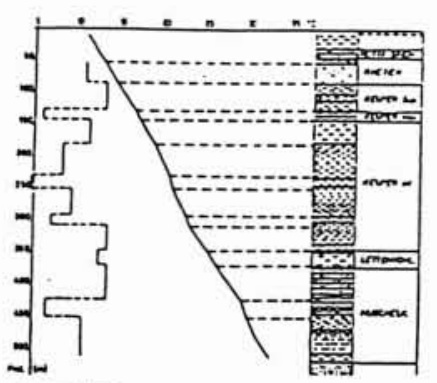

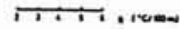

Figure 1. - Profil de température Cercueil 1 (R. Gable, 13.9.79).

(La ligne en pointillés correspond au gradient calculé pour chaque formation).

Figure 2. - Profil thermique enregistré dans le puits de Cergy (R. Gable, 1982).

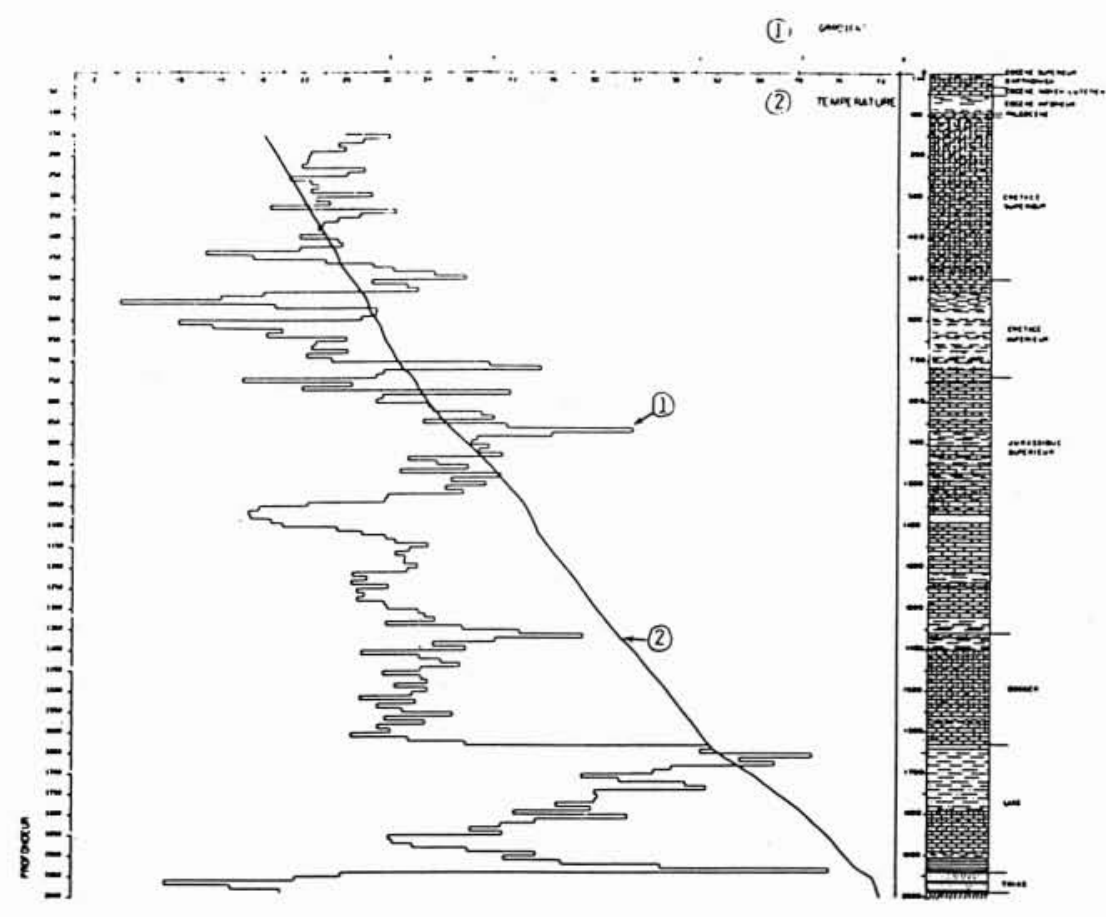

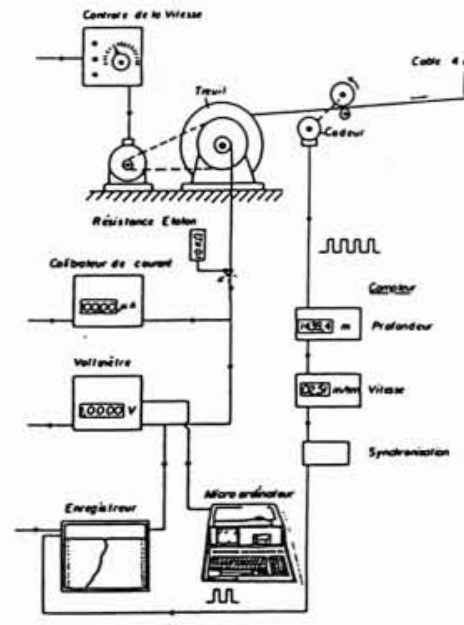

Figure 3. - Schéma de principe de fonctionnement de l'unité logging.
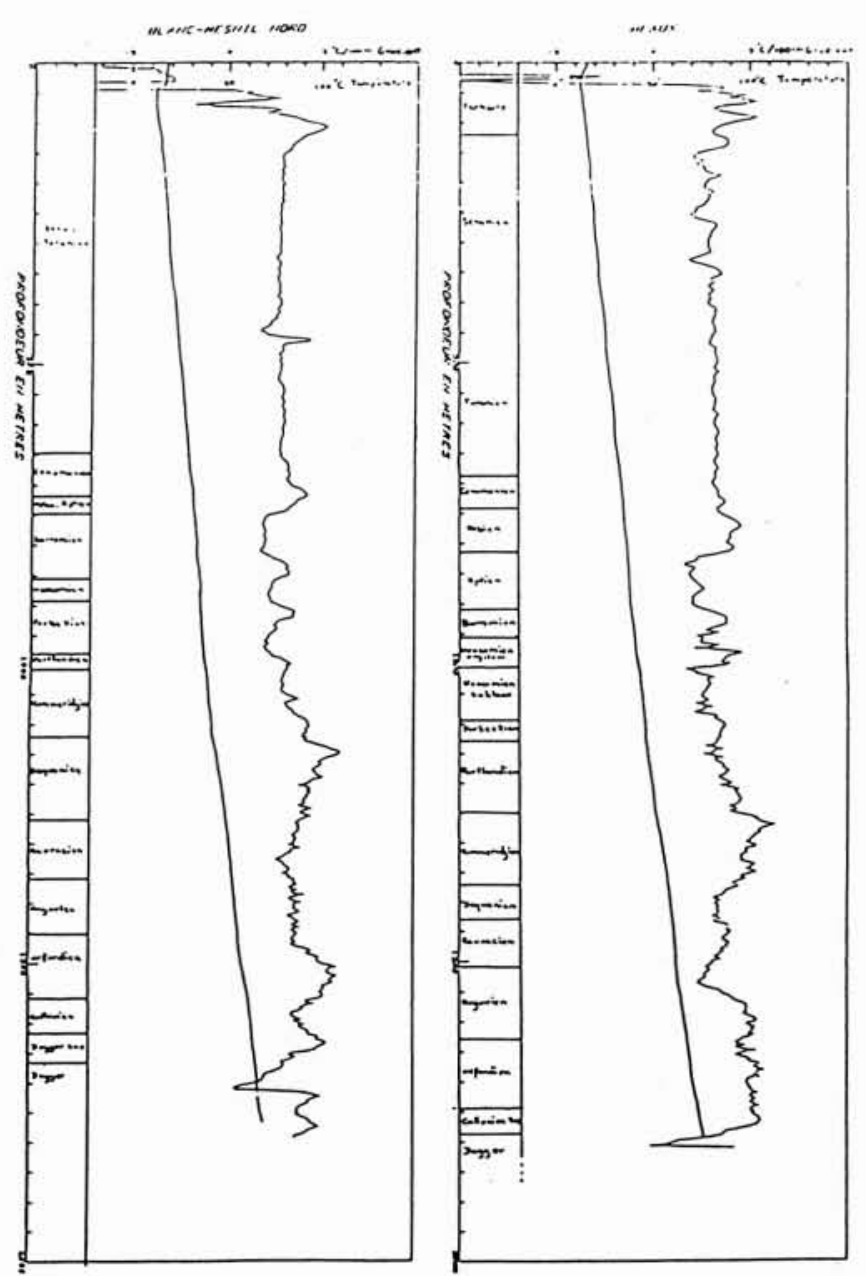

Figure 4. - Profils de température et gradient géothermique.

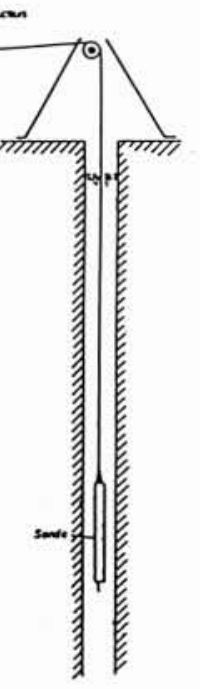




\section{Modélisation}

Il s'agit de définir des modèles de la répartition des températures dans le sous-sol.

Deux types d'approche peuvent être tentés :

- la méthode géostatistique permet à partir de valeurs expérimentales de donner l'estimation la meilleure possible du champ de température;

- les modèles conductifs, numériques, donnent une répartition théorique des températures à partir d'un certain nombre d'hypothèses. Dans la plupart des cas, on cherche le régime thermique stationnaire.

\subsection{Modélisation thermique}

Nous utilisons pour l'instant seulement des modèles bidimensionnels, ce qui suppose des structures cylindriques.

Des modèles 3D représenteraient peut être mieux la réalité géologique mais déjà très lourds à mettre en œuvre, ils nécessitent une très bonne connaissance de la répartition spatiale de nombreux paramètres tant géologiques que géophysiques : une telle information n'existe pas pour l'instant.

Il existe quelques solutions analytiques relativement simples de l'équation de la chaleur pour des modèles simples mais aux hypothèses souvent trop schématiques. On est conduit très vite à utiliser des modèles numériques discrets utilisant soit les différences finies, soit les éléments finis.

La méthode des éléments finis est l'une des méthodes les plus utilisées actuellement, cette méthode très générale s'applique à la majorité des problèmes rencontrés dans la pratique : problèmes stationnaires ou non, linéaires ou non, définis dans un domaine géométrique quelconque à une, deux ou trois dimensions. De plus, elle s'adapte très bien aux milieux hétérogènes.

Les méthodes de résolution de l'équation de chaleur par différences finies sont d'un emploi plus souple et nécessitent moins l'utilisation intensive de l'ordinateur. Mais on est assujetti aux conditions aux limites, à savoir :

- un flux latéral nul;

- un flux vertical et uniforme à la base;

- une température uniforme à la surface.

L'illustration suivante (Figure 5) montre un exemple d'isothermes calculées par modélisation théorique suivant une coupe Ouest-Est dans le Bassin parisien.

La distribution théorique calculée rend compte de la distribution des températures mesurées en forage, à l'équilibre.
Les contraintes retenues pour le modèle sont : modèle à 6 couches avec flux à la base $\Phi=90 \mathrm{mwm}^{-2}$ et température en surface $t=15^{\circ} \mathrm{C}$.

Les différentes conductivités thermiques choisies sont obtenues par mesures en laboratoire, interpolation ou approximation :

$\begin{array}{lll}\text { K1 } & \text { Crétacé sup. } & 3.2 \\ \text { K2 } & \text { Crétacé inf. } & 3.6 \\ \text { K3 } & \text { Jurassique sup. } & 2.5 \\ \text { K4 } & \text { Lias } & 1.8 \\ \text { K5 } & \text { Trias } & 2.5 \\ \text { K6 } & \text { Socle } & 4.5\end{array}$

\subsection{La modélisation géostatistique}

Tout d'abord mise au point et utilisée dans le domaine minier depuis plusieurs années, la géostatistique a été appliquée à différents domaines des sciences de la terre : recherche pétrolière, hydrogéologie et surtout météorologie. La théorie repose sur des méthodes qui allient l'aspect déterministe (dérives, tendances) et l'aspect aléatoire (irrégularités, fluctuations) des phénomènes naturels. Cet aspect aléatoire a néanmoins une structure que le variogramme qui quantifie la variabilité du phénomène entre 2 points donnés met en évidence. Il peut être déterminé à partir de mesures disponibles, ajusté à un modèle, et permet alors de définir un estimateur optimal de la variable en tout point où il n'y a pas de mesures. pour nous il s'agit de définir la distribution bidimensionnelle réelle ou probable du champ de température en tout point. Des contraintes sont imposées au modèle: contraintes thermique, géologique et structurale.

Les techniques de krigeage permettent l'estimation optimale de la température en tout point du sous-sol à partir des mesures directes ou indirectes obtenues dans les forages. Ici, ces techniques ont été adaptées pour prendre en compte l'accroissement régulier de la température avec la profondeur. La température est séparée en deux composantes :

- une dérive régulière, caractérisée par un gradient vertical, celui-ci est lié à la conductivité thermique;

- une fluctuation autour de cette dérive, caractérisée par son variogramme.

Dans une première étude, on a admis que la conductivité thermique pouvait être considérée comme constante à l'intérieur d'une formation géologique donnée, et on a 


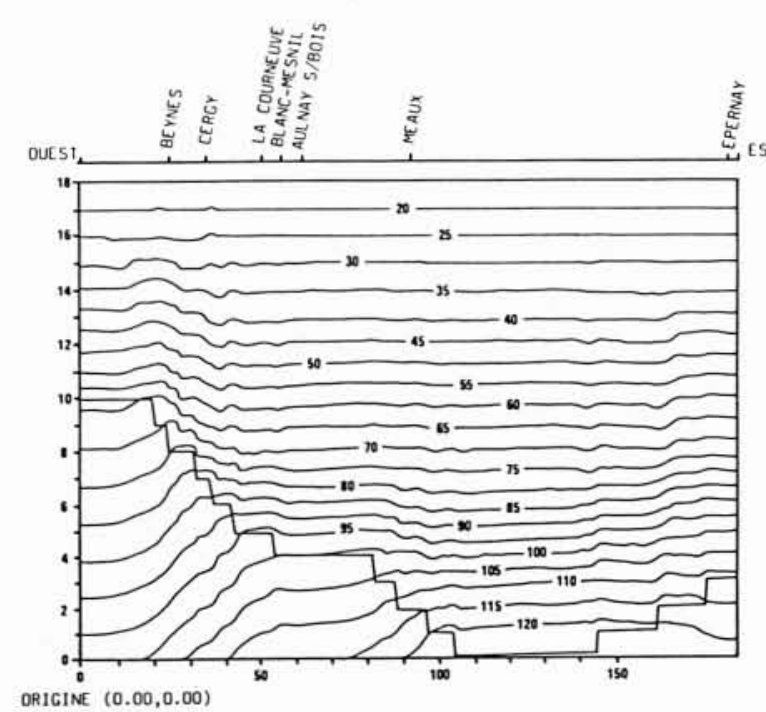

Figure 5. - Exemple d'isothermes calculées par modélisation théorique bidimensionnelle (coupe Bassin parisien E-W).

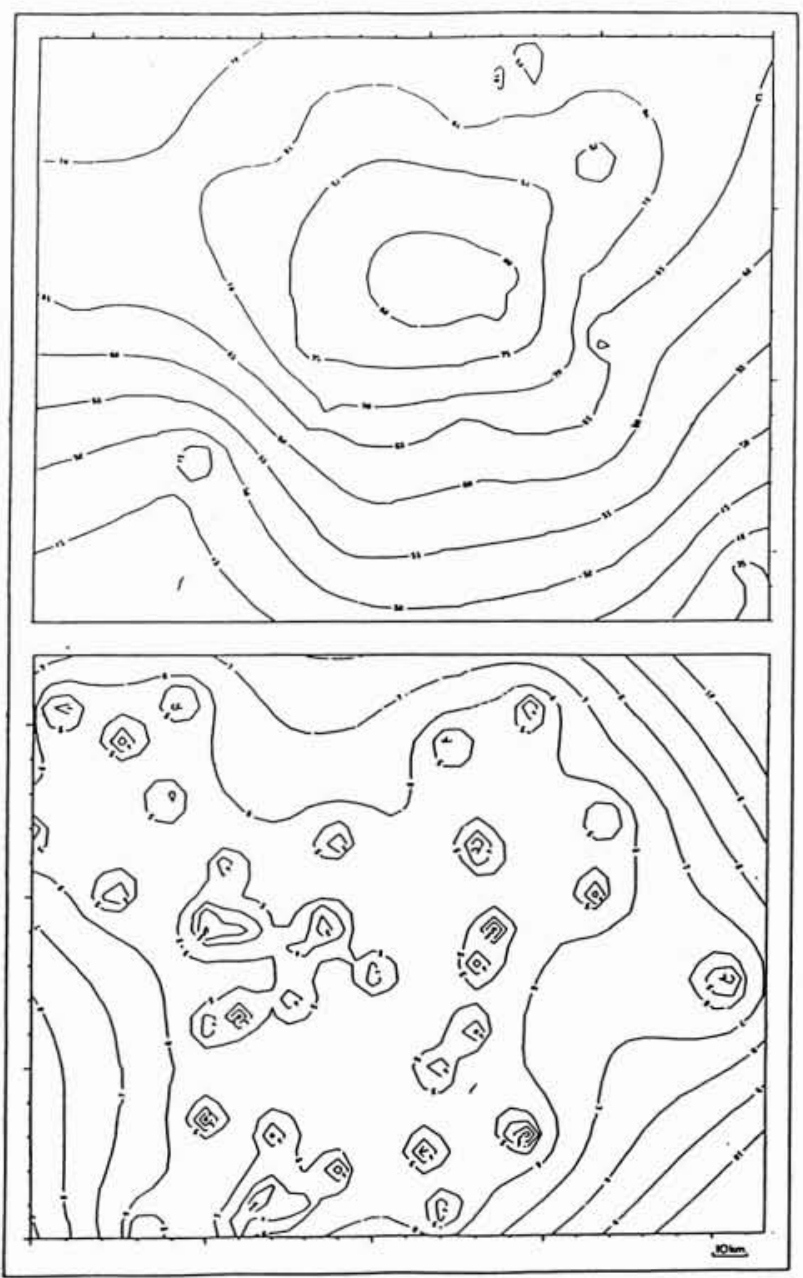

Figure 6. - Estimation de la température de l'aquifère du Dogger - Bassin parisien (R. Gable, P. Watremez, 1983). effectué le krigeage de la température en prenant en compte les toits des différentes formations géologiques. Les valeurs de la conductivité sont ajustées localement en fonction des données du voisinage. L'examen des variogrammes obtenus conduit aux observations suivantes :

- verticalement, une fois l'effet prépondérant de la dérive éliminé, les variations résiduelles peuvent atteindre $1^{\circ} \mathrm{C}$ en $100 \mathrm{~m}$, et $4^{\circ} \mathrm{C}$ en $500 \mathrm{~m}$ (risque $5 \%$ );

- horizontalement, on peut avoir des variations de température de $8{ }^{\circ} \mathrm{C}$ en quelques kilomètres (risque $5 \%$ ).

$\mathrm{Au}$ niveau du krigeage, dès qu'on s'éloigne des données, la précision de l'estimation n'est plus que de l'ordre de $\pm 10^{\circ} \mathrm{C}$. (exemple : estimation de la température de l'aquifère du Dogger - Bassin parisien, figure 6).

Cette étude est reprise actuellement. En effet, il s'avère sur les logs complets effectués récemment que le profil des conductivités thermiques est bien le même d'un forage à l'autre, mais que la conductivité ne peut pas être considérée comme constante dans chaque formation géologique. En tenant compte de l'allure réelle du profil des conductivités, on réduira l'amplitude des fluctuations résiduelles, et on améliorera d'autant la précision du krigeage.

\section{Références}

WAtremez Pierre (1980), - Flux de chaleur sur le Massif Armoricain et sur la marge continentale : essai de modélisation de l'évolution thermique de la marge armoricaine. Thèse $3^{e}$ cycle Univ. Brest.

Chiles Jean-Paul, Gable Robert (1984). - Three-dimensional modeling of a geothermal field. Geost. for National Ressources Charact. Part 2, 587-598. D. Reidel Publ. Company.

GABLE Robert (1982). - mesures, analyses et interprétation de profils thermiques. Etude BRGM 82 SGN 413.

GABLE Robert (1984). - Acquisition et interprétation des données géothermiques. Thèse de Doctorat d'Etat, Univ. Paris VI. 
L'ensemble des discussions qui se sont développées à la suite des divers exposés de cette session fait apparaître globalement deux types de préoccupations de la part des intervenants : - la connaissance précise des paramètres conditionnant les phénomènes de transfert de chaleur et d'humidité dans le sol et en surface,

- l'aptitude des modèles théoriques présentés à prévoir la réalité physique.

Monsieur GOLDSTEIN pose en premier lieu le problème de la détermination des coefficients de diffusion pris en compte au cours de simulations numériques du comportement thermique et hydrique de sols, présentées dans le cadre d'études développées à l'Institut de mécanique des fluides de Toulouse. Monsieur BORIES précise qu'en ce qui concerne la conductivité thermique, ce paramètre a été déterminé à l'aide d'une méthode expérimentale de régimes stationnaires. Les coefficients de diffusion de masse ont été, également, évalués par des expériences spécifiques, en admettant un découplage au niveau de la quantité de mouvement entre les écoulements en phase gazeuse et en phase liquide.

La présentation de relevés de températures dans un bassin sédimentaire effectuée ensuite par Monsieur WATREMEZ, soulève, entre autres, le problème de la précision des mesures in situ par forage : risque de convection autour du point de mesure (Monsieur BORIES), perturbation de la mesure par le forage lui-même (Monsieur BANAL). D'autre part, le champ de température dans le sol peut être également influencé par l'existence d'incidents de structure (batholites) d'après Monsieur GRJEBINE, ou par la présence dans les fractures, de régimes de convection naturelle (Monsieur WATREMEZ) induisant des inversions de gradients de température, soulignées par Monsieur BORIES. Les variations du paramètre conductivité thermique (de 1 à 5) utilisé dans les modèles thermiques du Bassin Parisien, paraissent élevées à Monsieur FIRDAOUSS. Monsieur WATREMEZ fait alors remarquer que ces variations, obtenues par des mesures effectuées à faible profondeur ou en laboratoire, sont normales, en raison de la présence de fluides dans les roches qui modifient sensiblement la conductivité thermique équivalente.

La représentativité des modèles théoriques utilisés et des hypothèses formulées, en vue de simuler les champs de température et d'humidité dans les sols, apparaît ensuite comme le thème principal des discussions.

En ce sens, le concept de similitude, introduit dans la modélisation de la variabilité spatiale de paramètres macroscopiques (perméabilité, conductivité, etc.) par l'exposé de Monsieur BRUNET, soulève une question de Monsieur QUINTARD quant à la justification de pratiquer la similitude sur des grandeurs macroscopiques faisant elles-mêmes intervenir des couplages de phénomènes microscopiques ayant des similitudes différentes. Monsieur BRUNET reconnaît que si cette approche implique de fortes hypothèses, elle fournit en général une bonne adéquation entre modèles et mesures, dans le cas de milieux poreux à granulométrie peu étendue (exemple des sols de type "sable "); par contre, lorsque les tailles de particules sont très différentes, la similitude n'est plus, effectivement, aussi bonne. Monsieur GRJEBINE pose le problème important du rôle joué par la couverture biologique dans la modulation des phénomènes de transfert sol-atmosphère, particulièrement lors de l'existence de mécanismes de surface séquentiels, tels que effets de pluie et évaporation de l'humidité, se traduisant par des apports ou des pertes de calories. Monsieur BRUNET souligne que, dans le cas de sols nus, des gradients thermiques très importants peuvent exister dans les couches superficielles à l'échelle de la journée, alors qu'en présence d'un couvert végétal, ils seront en moyenne beaucoup plus modérés. A l'échelle de l'année, la différence est beaucoup moins sensible au niveau du stockage de chaleur, les températures évoluant de manière semblable en profondeur. Il semble donc, comme le fait remarquer Monsieur le Président, que la prise en compte de phénomènes de type biologique dans les modèles prévisionnels de transfert permettrait une approche plus précise de la réalité, en particulier lors des opérations (sensiblement différentes) de séchage de grains de nature biologique ou minérale.

Monsieur BENET, après avoir décrit une modélisation mathématique locale des sols non saturés, basée sur la Thermodynamique des Processus Irréversibles, présente des résulats expérimentaux sur le comportement thermique et hydrique de la couche d'un sol située au-dessus d'un stockage de chaleur. Monsieur GREEBINE s'interroge à nouveau quant à l'incidence sur ces résultats de la présence en surface d'une couche imperméable à la vapeur. Monsieur BENET répond que dans la configuration expérimentale étudiée, les transferts d'énergie en phase gazeuse, et également en phase liquide sont négligeables. Monsieur BORIES fait alors remarquer qu'après les exposés de MM. BRUNET et BENET on peut finalement s'interroger quant à l'intérêt d'avoir recours à des modèles compliqués, quand on se rend compte que dans les deux cas, on arrive à rendre compte de certaines expériences avec des simplifications draconniennes. Il précise qu'en l'occurence les deux cas présentés ne sauraient avoir valeur de règle générale et qu'il convient de procéder à des analyses de sensibilité aux paramètres avant toute simplification a priori. Des travaux effectués à Toulouse par MM. RECAN et CRAUSSE illustrent d'ailleurs parfaitement les risques auxquels peuvent conduire ces réductions de modèles dans l'étude des transferts couplés en milieu poreux. Monsieur BRUNET souligne qu'on ne peut parler d'un modèle qu'en fonction des objectifs qu'on lui assigne. Monsieur BENET précise alors qu'il faut quand même distinguer les modèles permettant une analyse fine de la physique des phénomènes et des modèles de prévision liés à une application.

Monsieur le Président conclut en développant l'idée que le modèle général est élaboré pour aborder toute situation, puis on le simplifie, compte tenu des variables qui paraissent essentielles dans la configuration physique étudiée.

Nota: Le texte ci-dessus fait la synthèse des discussions qui ont suivi la présentation des quatre communications présentées le 14 novembre 1984 sur "les transferts thermiques dans le sous-sol ". Cette synthèse a été préparée par M. P. Crausse, Maitre de Conférences à l'INP de Toulouse. concernées.

Pour la commodité des lecteurs, nous la présentons à la suite de chacune des quatre communications 